\title{
Skeletal Muscle Neoplasm
}

National Cancer Institute

\section{Source}

National Cancer Institute. Skeletal Muscle Neoplasm. NCI Thesaurus. Code C6514.

A benign or malignant mesenchymal neoplasm arising from skeletal muscle. 